1 Universidade Federal de São Carlos (UFSCar), Programa de PósGraduação em Gestão da Clínica - São Carlos (SP), Brasil.

laisvmagri@gmail.com

2 Universidade Federal de São Carlos (UFSCar),

Programa de Pós-Graduação em Gestão da Clínica - São Carlos (SP), Brasil.

geovani.gurgel@gmail.com

3 Universidade Estadual de Campinas (Unicamp) Faculdade de Odontologia de Piracicaba (FOP)

Departamento de Odontologia Social Piracicaba (SP), Brasil. ferdruva@gmail.com

4 Universidade Estadual Paulista (Unesp), Faculdade de Odontologia de Araraquara, Departamento de Odontologia Social Araraquara (SP), Brasil. epstag@gmail.com

5 Universidade de São Paulo (USP), Faculdade de Medicina de Ribeirão Preto, Departamento de Medicina Social - Ribeirão Preto (SP), Brasil.

lucasgasparribeiro@gmail.com

\section{Estudo comparativo de indicadores de saúde bucal em município do estado de São Paulo}

\author{
Comparative study of oral health indicators in primary care of a São \\ Paulo State's city
}

Laís Valencise Magri1, Geovani Gurgel Aciole², Fernanda Gonçalves Dutra Salomão $\mathbf{3}$, Elaine

Pereira da Silva Tagliaferro ${ }^{\mathbf{4}}$, Lucas Gaspar Ribeiro ${ }^{\mathbf{5}}$

RESUMO Comparar indicadores de saúde bucal (Rendimento, Atrição, Ênfase em Prevenção Modificado, Relação Restauração/Extração) e Relação $1{ }^{a}$ Consulta/Urgência entre 15 Unidades de Saúde da Família e 11 Unidades Básicas de Saúde de município do interior paulista, de 2008 a 2011. Os dados foram coletados a partir do Sistema de Informações Ambulatoriais do SUS (SIA-SUS) e comparados os modelos de atenção por meio de série histórica, teste não paramétrico (Mann-Whitney) e estatística descritiva. Embora haja o processo de fortalecimento da Estratégia Saúde da Família o modelo assistencial representado pelas Unidades Básica de Saúde se mostra mais efetivo em alguns aspectos da saúde bucal no serviço público.

PALAVRAS-CHAVE Odontologia comunitária; Indicadores básicos de saúde; Sistemas de informação; Serviços de saúde bucal; Atenção Primária à Saúde.

ABSTRACT To compare four oral health indicators (Income, Attrition, Modified Prevention Emphasis, Restoration/Extraction) and the 1st Consultation/Urgency Relationship in 15 Family Health Units and 11 Basic Health Units, in an interior city of São Paulo State, 2008-2011. The data were collected from SIA-SUS and the indicators compared through historical series using the non-parametric test (Mann Whitney) and descriptive statistics. Although there is a strengthening process of the Family Health Strategy, the care model represented by the Basic Health Units still proves more effective in some aspects of oral health in public service.

KEYWORDS Community dentistry; Health status indicators; Information systems; Dental health services; Primary Health Care. 


\section{Introdução}

A Atenção Primária à Saúde (APS) tem papel fundamental na estruturação dos sistemas de saúde na medida em que representa a principal 'porta de entrada' dos usuários a todos os níveis de cuidado: primário, secundário e terciário. Assim, representa o alicerce de toda a rede de saúde sendo responsável pela coordenação do cuidado e resolubilidade da grande maioria das demandas de usuários que buscam atendimento no Sistema Único de Saúde (SUS).

Dois modelos assistenciais à saúde coexistem no âmbito da APS, representados pelas Unidades de Saúde da Família (USF) e Unidades Básicas de Saúde (UBS). Nas USF o trabalho é desenvolvido por uma equipe de referência com formação generalista, que pode contar com o apoio matricial e que atua com base no diagnóstico da população adscrita, compreendendo cobertura populacional de no máximo 1.000 famílias. As UBS, por sua vez, possuem uma equipe com médicos especialistas e suas ações geralmente não estão baseadas no diagnóstico do território, além da cobertura populacional exceder sua capacidade de atendimento. Ambos os modelos têm como filosofia a promoção da saúde e devem produzir indicadores que avaliem a quantidade e a qualidade das ações em saúde.

$\mathrm{Na}$ área de saúde bucal, até a década de 1990 as ações se constituíam de forma paralela e afastada do processo de organização dos demais serviços de saúde. A partir de 1994, as ações passaram a ser integradas com as demais áreas visando prevenir doenças e promover saúde. Atualmente, a utilização de Indicadores de Saúde Bucal (ISB) tem possibilitado à odontologia, no SUS, experimentar uma prática planejada a partir do diagnóstico territorial e de avaliações periódicas. A maioria dos ISB é encontrada no Sistema de Informações Ambulatoriais (SIA) da APS (CARNUT; FIGUEIREDO; GOES, 2010). Em 2005, a proposição de uma Política Nacional de Saúde Bucal, chamada de Brasil Sorridente, visa impulsionar a mudança da lógica assistencial do setor na direção de um modelo centrado na saúde bucal coletiva e em práticas de promoção e prevenção com vistas a diminuir o impacto das práticas curativas e mutiladoras (AQUILANTE; ACIOLE, 2015).

Entretanto, pouco tem sido estudado a respeito do assunto devido à grande dificuldade para a incorporação da prática de registro de informações nos serviços de saúde, bem como à falta de planejamento e avaliação no desenvolvimento de ações voltadas para a saúde bucal (LOIVOS ET AL., 2006). O objetivo deste estudo foi analisar comparativamente ISB da APS entre USF e UBS de um município do interior paulista por meio de série histórica, a partir de dados obtidos do Sistema de Informações Ambulatoriais do SUS (SIA-SUS).

\section{Material e métodos}

Trata-se de estudo observacional descritivo de corte transversal no qual foram coletadas informações secundárias provenientes do banco de dados do SIA-SUS. O estudo foi desenvolvido segundo os princípios do Comitê de Ética em Pesquisa da Universidade Federal de São Carlos (UFSCar), sob o protocolo $\mathrm{n}^{\circ}$. 01166712.0.0000.5504. Houve dispensa do Termo de Consentimento Livre e Esclarecido (TCLE) devido à utilização de base de dados secundária (Sistema de Informação de domínio público), sem qualquer identificação de usuários ou de equipes incluídos no estudo. A única informação disponibilizada concerne ao tipo de modelo assistencial (USF ou UBS), sem a identificação de unidade e/ou profissionais integrantes das Equipes de Saúde Bucal (EqSB).

Esta pesquisa foi realizada no município de São Carlos (SP), que é considerado de grande porte e que, em relação à saúde bucal, conta com 17 USF e 12 UBS, das quais apenas duas não possuem consultório odontológico; além de um Centro de Especialidades Odontológicas (CEO). Este município é credenciado no Programa Brasil Sorridente desde 2006. Os critérios de inclusão utilizados foram 
a presença de consultório odontológico na Unidade de Saúde (US) com inauguração antes de 2010, totalizando 15 USF e 11 UBS. Os dados odontológicos produzidos entre 2008 e 2011 foram coletados a partir do SIA-SUS, com base no Boletim de Produção Ambulatorial (BPA), que traz a produção mensal em termos de procedimentos odontológicos de cada US do município.

Com os dados obtidos foram calculados os ISB propostos por Narvai (1996), a saber:

a) Indicador de Rendimento (IR), que expressa a capacidade produtiva da hora do Cirurgião-Dentista (CD) na assistência em termos de quantidade de unidade de trabalho que a compõe, considerando-se 160 horas mensais para USF e 240 horas mensais para UBS;

b) Indicador de Atrição (IA), referente ao valor percentual de quanto da população teve acesso a tratamento odontológico e efetivamente realizou todo o tratamento. Valores inferiores a $90 \%$ indicam dificuldades para atingir a população alvo;

c) Indicador Relação Restauração/Extração (IRRE), que mensura a orientação das ações para a reabilitação ou para a mutilação. Valores menores que um indicam uma prática mutiladora;

d) Indicador Ênfase em Prevenção Modificado (IEPM), que compara a proporção de procedimentos preventivos versus curativos a fim de monitorar o modelo de atenção. $\mathrm{O}$ esperado é que os valores sejam próximos de um, o que representaria equilíbrio entre as práticas preventiva e curativa no modelo assistencial.

Dentre os ISB estudados, os três primeiros avaliam efetividade (IR, IA, IRRE) e o último avalia o modelo de atenção (IEPM).

Também se calculou e incluiu no estudo a Relação $1^{\text {a }}$ Consulta/Urgência (RPCU) devido à sua importância, pois embora não seja considerada um ISB avalia o acesso ao tratamento odontológico em comparação com os atendimentos odontológicos de urgência (usuários que não tiveram acesso ao tratamento odontológico). Os dados produzidos pelas USF e pelas UBS foram comparados por meio de série histórica, utilizando-se o teste não paramétrico (Mann-Whitney) e estatística descritiva, adotando-se como nível de significância 5\% (Bioestat 5.0).

\section{Resultados}

A tabela 1 sintetiza os cinco itens de avaliação utilizados na pesquisa e apresenta os dados relativos à média, desvio-padrão, mediana, mínimo e máximo para cada ISB e RPCU. Foi encontrada significância estatística $(\mathrm{p}<0.05)$ na comparação USF/UBS apenas para o IRRE e RPCU, sendo que os melhores resultados foram observados no modelo assistencial UBS.

Tabela 1. Medidas de frequência e dispersão (desvio-padrão; mediana; mínimo; máximo) dos Indicadores de Saúde Bucal (ISB) segundo o tipo de unidade de saúde da atenção primária do município de São Carlos (SP), 2008-2011

\begin{tabular}{|c|c|c|c|}
\hline \multirow{2}{*}{ Indicadores } & \multicolumn{2}{|c|}{ Tipo de Unidade } & \multirow{2}{*}{ p-valor ${ }^{\star}$} \\
\hline & USF & UBS & \\
\hline Indicador de Rendimento & $18,2(12,2 ; 16 ; 0 ; 75)$ & $20(10,9 ; 20 ; 0 ; 58)$ & 0,20 \\
\hline Indicador Ênfase em Prevenção Modificado & $0,12(0,33 ; 0 ; 0 ; 1)$ & $0,06(0,24 ; 0 ; 0 ; 1)$ & 0,59 \\
\hline Indicador de Atrição & $0,13(0,20 ; 0,15 ; 0 ; 0,68)$ & $0,07(0,17 ; 0 ; 0 ; 0,77)$ & 0,17 \\
\hline Indicador Relação Restauração/Extração & $4,7(2,1 ; 4 ; 2 ; 14)$ & $5,2(1,4 ; 5 ; 0 ; 8)$ & $0,016^{\star \star}$ \\
\hline Relação 1a Consulta/Urgência & $0,3(0,5 ; 0 ; 0 ; 2)$ & $0,7(0,6 ; 1 ; 0 ; 2)$ & $0,002^{\star \star}$ \\
\hline
\end{tabular}


Para o IEPM o valor médio anual encontrado nas USF $(0,12)$ foi o dobro do encontrado nas UBS $(0,06)$. Relação semelhante foi observada para o IA com relação às USF $(0,13)$ e UBS $(0,07)$, embora ambos os ISB sem significância estatística.

$\mathrm{O}$ valor médio encontrado para o Indicador de Rendimento foi de 18,2 para USF e 20 para UBS (gráfico 1), demonstrando que a capacidade produtiva dos profissionais CDs totalizou 19 procedimentos por hora trabalhada (em média). Por meio do gráfico 1 é possível verificar que, com a progressão do tempo, houve um aumento dos valores médios anuais do IR para as USF, que passou de 8,7 em 2008 para 26,5 em 2011, um aumento de $204 \%$. Já na UBS não houve alteração significativa, pois em 2008 o valor médio era de 18,3 e em 2011 de 19,9 .

Gráfico 1. Médias anuais do Indicador de Rendimento dos modelos assistenciais da atenção básica de São Carlos (SP), 2008 a 2011

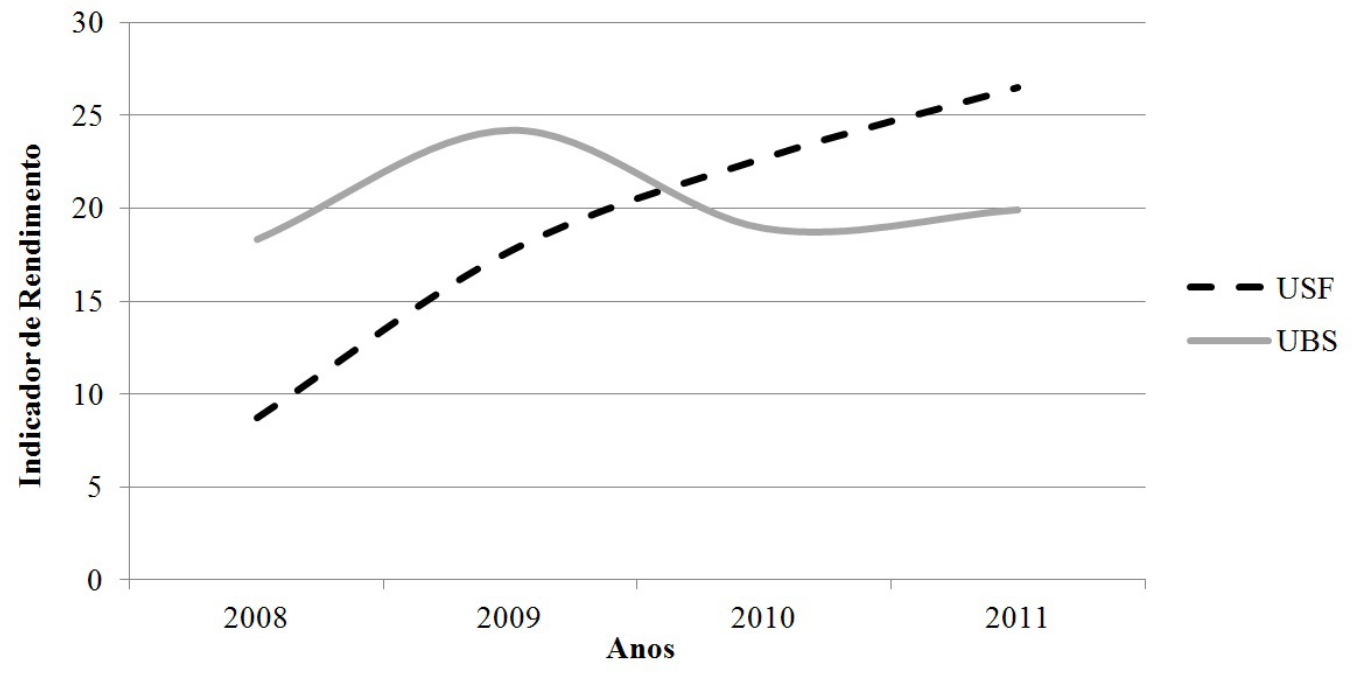

Fonte: Elaboração própria.

Os valores médios anuais do IEPM encontrados para as USF e UBS foram de $0,12 \mathrm{e}$ 0,06 , respectivamente, ambos ainda bastante insatisfatórios. É importante considerar que este indicador mostrou-se maior nas USF quando comparado com as UBS (dobro do valor) em praticamente todo o período estudado, com exceção do ano 2009, conforme gráfico 2. De maneira geral, em ambos os modelos houve redução nos valores médios anuais destes indicadores entre 2008 e 2011: nas USF reduziu de 0,68 para 0,46 enquanto que nas UBS os valores decaíram de 0,45 para 0,37 . 
Gráfico 2. Médias anuais do Indicador Ênfase em Prevenção dos modelos assistenciais da atenção básica de São Carlos (SP), 2008 a 2011

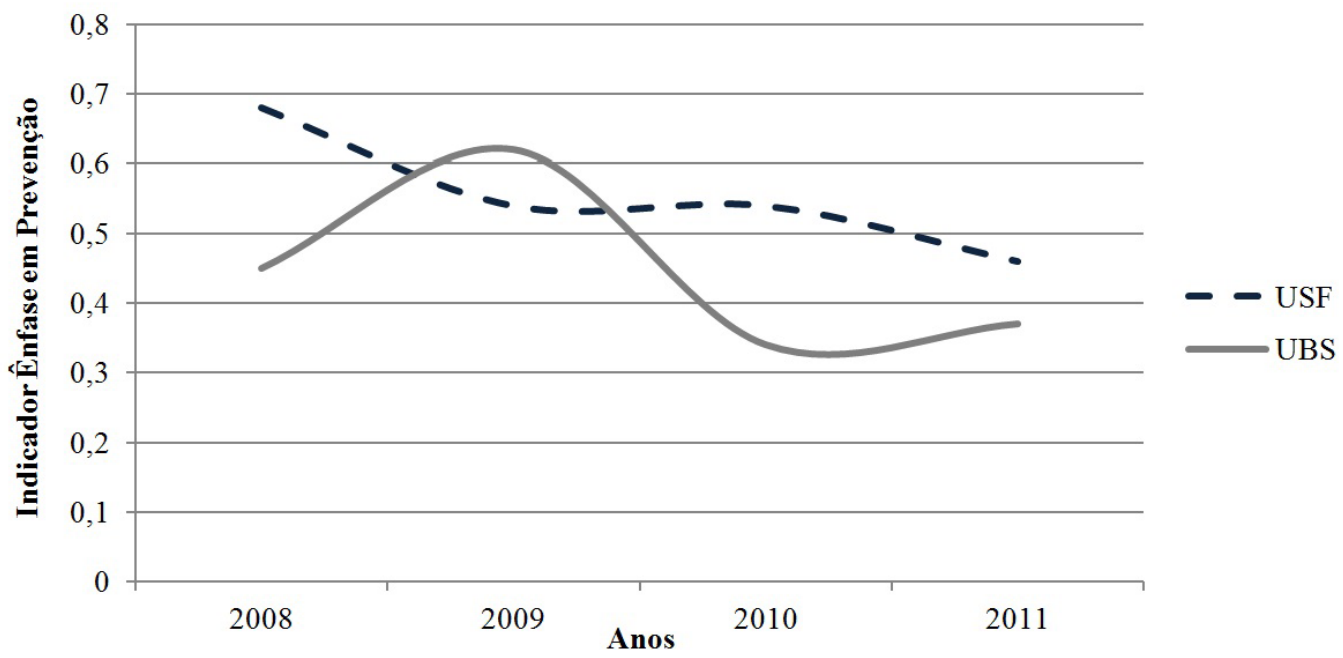

Fonte: Elaboração própria.

Por meio do gráfico 3 é possível verificar que as médias anuais do IA são bastante insatisfatórias. As USF apresentaram ao longo dos anos as médias de 0,39 (2008); 0,06 (2009); 0,02 (2010) e 0,01 (2011). Nas UBS as médias foram de 0,09 (2008); 0,11 (2009); 0,04 (2010) e 0,03 (2011), ou seja, em ambos os modelos houve redução deste indicador ao longo dos anos, queda que se mostrou mais acentuada nas USF.

Gráfico 3. Médias anuais do Indicador de Atrição dos modelos assistenciais da atenção básica de São Carlos (SP), 2008 a 2011

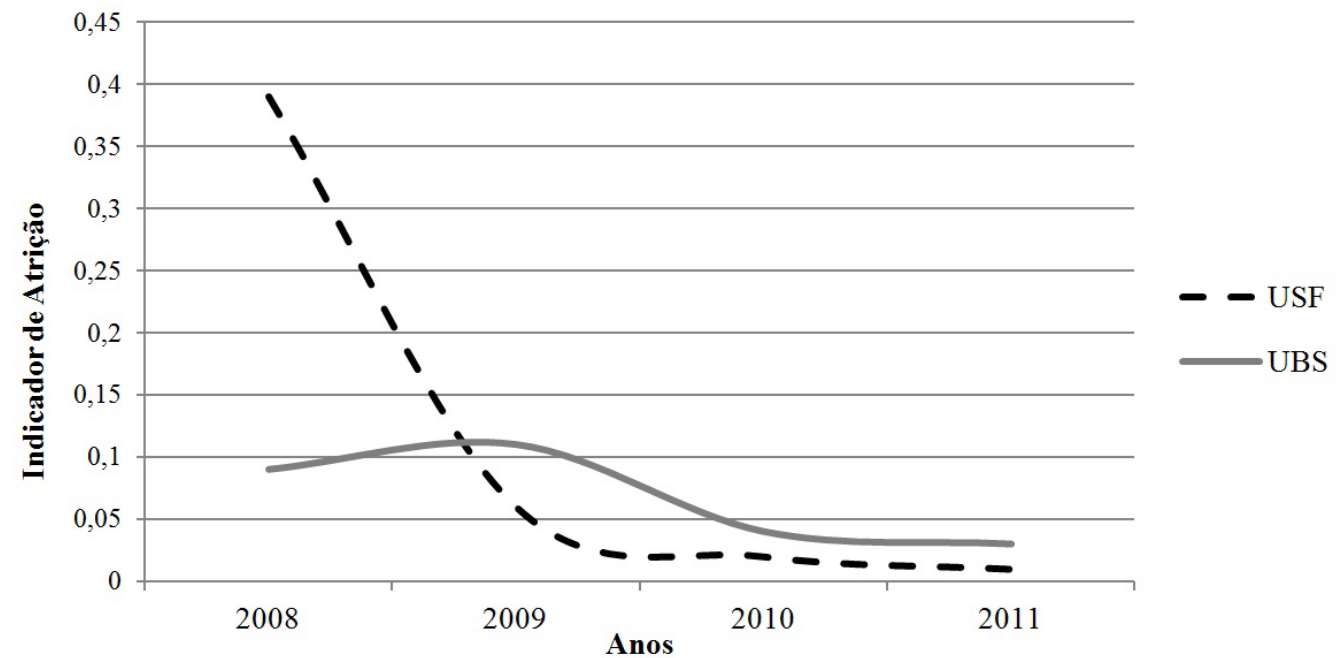


Em 2008, o valor médio do IRRE era de 4,53 para as USF e de 4,31 para as UBS; já em 2011 o valor médio deste indicador para as USF subiu para 7,03 (aumento de 63\%) enquanto que para as UBS o valor encontrado foi de 6,48 (aumento de $50 \%$ ). Por meio do gráfico 4 é possível observar o aumento deste indicador em função da série histórica. Mesmo os valores sendo maiores nas UBS, com significância estatística quando comparados às USF, nestas, contudo, a proporção do aumento em função do tempo foi superior, sendo que em 2011 o valor do indicador mostrou-se superior nas USF quando comparadas às UBS.

Gráfico 4. Médias anuais do Indicador Relação Restauração/Extração dos modelos assistenciais da atenção básica de São Carlos (SP), 2008 a 2011

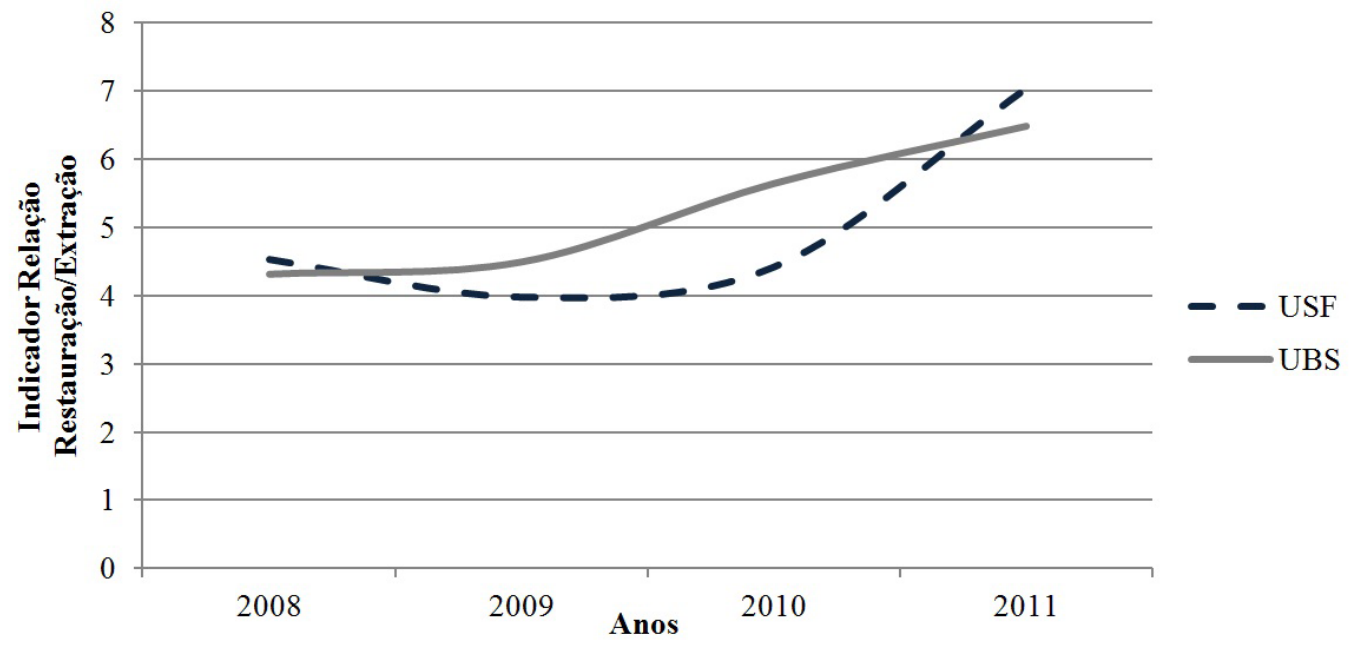

Fonte: Elaboração própria.

Para a RPCU, o $p$ encontrado foi de 0,002, o que denota forte significância estatística na comparação USF/UBS, havendo superioridade para as UBS. Os resultados encontrados podem sugerir que as UBS garantem maior acesso ao tratamento odontológico do que aos serviços odontológicos de urgência e emergência quando comparadas às USF. Nos quatro anos pesquisados as médias anuais desta relação foram superiores para as UBS, de modo que as USF obtiveram as seguintes médias na série histórica: 0,52 (2008); 0,83 (2009); 0,97 (2010) e 0,86 (2011), ou seja, em nenhum momento do estudo foi atingida a relação 1, o que sugere que na Estratégia Saúde da Família (ESF) há predomínio de atendimentos odontológicos de urgência.
Houve aumento desta relação para as UBS, que em 2008 apresentavam média em torno de 1 e, em 2011, diante de um aumento de $50 \%$, a média observada foi 1,5 .

\section{Discussão}

Os ISB são de suma importância para a gestão dos serviços de saúde, pois possibilitam uma visão ampla do que foi produzido em termos de procedimentos e atendimentos odontológicos. Neste sentido, os Sistemas de Informação (SI) assumem grande importância na medida em que representam a fonte de informações para a obtenção dos indicadores (BARROS; CHAVES, 2003; PEREIRA ET AL., 2012). 
O SIA compreende atualmente o SI mais utilizado no âmbito do SUS e possui informações que extrapolam a esfera financeira, constituindo-se uma importante ferramenta para a gestão dos serviços de saúde. Trata-se do SI mais indicado para a obtenção dos ISB, pois o Sistema de Informação da Atenção Básica (Siab) ainda passa por um processo de fortalecimento que lhe viabiliza a utilização na ESF, não podendo ser aplicado para as UBS (VOLPATO; SCATENA, 2006).

Neste estudo, que comparou alguns ISB da APS de um município do interior paulista, foi evidenciada superioridade das UBS em relação à USF $(\mathrm{p}<0.05)$ para o IRRE e para a RPCU. Estes resultados demonstram que as UBS estão mais estruturadas em seu processo de trabalho do que as USF, que são unidades de saúde mais novas e ainda em estabelecimento e construção de suas equipes. Todavia, há que se considerar uma tendência de fortalecimento da ESF no município estudado, pois em todos os indicadores, com exceção da RPCU, houve melhora significativa em função da série histórica, sendo que em 2011 os valores destes indicadores nas USF estavam superiores ou muito próximos em comparação com as UBS.

Em relação ao IEPM, os resultados encontrados neste trabalho vão ao encontro de outros trabalhos realizados no Brasil (BARROS; CHAVES, 2003; VOLPATO; SCATENA, 2006; CELESTE ET AL., 2011). A relação ações preventivo/curativas ainda está longe do esperado, contudo há uma evidente superioridade da ESF que, mesmo lentamente, vem promovendo mudanças na odontologia a nível público no Brasil.

Estudos desenvolvidos nas regiões Norte e Nordeste do Brasil, que abordam os impactos das EqSB no acesso aos serviços odontológicos, também têm apontado para um fortalecimento destas ações após a incorporação da saúde bucal na ESF (EMMI; BARROSO, 2008; ROCHA; GOES, 2008). Adicionalmente, outros dois estudos avaliaram as séries temporais anuais de procedimentos odontológicos realizados no SUS em três municípios brasileiros e encontraram grande variação nas taxas em função dos anos. Estes estudos não trazem conclusões sobre a existência de tendências temporais na saúde bucal pública brasileira (BARROS; CHAVES, 2003; VOLPATO; SCATENA, 2006).

Os resultados deste estudo mostraram que não houve significância estatística para o IR na comparação entre os modelos assistenciais $(\mathrm{p}=0,20)$. Nos dois primeiro anos $(2008 / 2009)$, os valores deste indicador foram consideravelmente maiores nas UBS, todavia é necessário considerar que a maioria das USF do município foi criada a partir de 2005 , portanto ainda eram bastante recentes neste biênio. De 2008 a 2011 houve um aumento de $204 \%$ no valor médio do IR nas USF, tal resultado sugere que neste período houve uma tendência de fortalecimento da saúde bucal nas USF.

Um fato bastante relevante encontrado nos dados obtidos é o fenômeno de sazonalidade das ações preventivas, pois em ambos os modelos foi verificado que apenas em determinados meses do ano se alcançou valores considerados satisfatórios (considerando o IEPM), isto pode sugerir que as EqSB da APS desempenham ações de prevenção em períodos específicos do ano (por exemplo, em escolas e/ou exames epidemiológicos), não havendo, portanto, um equilíbrio entre as ações preventivas e curativas nos demais períodos do ano.

Este padrão sazonal de distribuição das ações preventivas também foi observado para quatro das cinco regiões brasileiras no estudo de Celeste et al. (2011). Segundo os autores, estes padrões cíclicos estão relacionados com vários aspectos, dentre eles a organização dos serviços de saúde (férias anuais dos CDs, por exemplo) e a demanda de períodos escolares, para citar uma possibilidade.

Esta concentração de ações preventivas se reflete também no indicador IEPM, que demonstrou resultados abaixo do esperado. 
Isto pode sugerir que a odontologia da APS deste município do interior paulista ainda se encontra voltada para a prática curativista e de reparo de danos.

No estudo realizado por Fernandes e Peres (2005) foi encontrado resultado parecido. A razão de procedimentos odontológicos coletivos na população entre 0 a 14 anos foi de 0,37 . Considerando que esta população geralmente é a mais contemplada pelas ações coletivas de prevenção, é possível afirmar que em outras regiões do País a saúde bucal também se encontra mais voltada para práticas curativas do que preventivas.

Diversos estudos da literatura científica apontam para a importância da ESF na reorientação do modelo assistencial. Embora haja grandes expectativas de resultados com esta estratégia, na prática isto é mais complicado do que parece. Souza e Roncalli (2007) realizaram um estudo com o objetivo de avaliar a incorporação da saúde bucal na ESF no estado do Rio Grande do Norte, onde a maioria dos municípios apresentou pouco ou nenhum avanço no modelo assistencial em saúde bucal. Os autores apontam para a necessidade de criação de políticas públicas que contemplem particularidades além do setor da saúde.

De fato, para que mudanças efetivas no modelo assistencial odontológico sejam possíveis, é de suma importância que haja um excelente planejamento das ações que serão desenvolvidas, bem como uma avaliação capaz de mensurar os resultados alcançados. Através da avaliação do IR e do IEPM é possível verificar que o município estudado vem aumentando a sua produção em termos de atendimentos odontológicos na APS, embora estes atendimentos provavelmente estejam direcionados para práticas assistenciais e curativista, pois as ações de prevenção e promoção da saúde (individuais ou coletivas) estão sendo deixadas de lado, de acordo com os valores obtidos para o IEPM.

Com relação ao IA, conforme indicado por
Narvai (1996), valores abaixo de 0,9 indicam dificuldade para atingir a população-alvo. Apesar de em ambos os modelos assistenciais os resultados encontrados terem sido abaixo do esperado, a média relativa aos 4 anos foi superior nas USF $(0,13)$ quando comparada à UBS $(0,07)$. Valores 0 foram observados com muita frequência (52\% para as UBS e $38 \%$ para as USF). Estes resultados demonstram que, embora sem significância estatística, a ESF consegue finalizar mais tratamentos odontológicos garantindo, desta maneira, mais acesso aos seus usuários.

Rocha e Goes (2008) publicaram um estudo que contrapõe estes resultados. Eles avaliaram os fatores associados ao acesso aos serviços odontológicos em Campina Grande (PB), comparando áreas cobertas e não cobertas pela ESF. Verificou-se que não há associação entre residir em uma área coberta pela ESF ou não coberta com o acesso aos serviços de saúde bucal. Os usuários que residiam em áreas não cobertas pela $\mathrm{ESF}$ tiveram 1,5 vezes mais chances de ter acesso a serviços de saúde bucal do que usuários que residiam em áreas de cobertura da ESF.

Considerando que o IA é um indicador de efetividade, é possível afirmar que as unidades de saúde da APS do município estudado não têm sido efetivas em suas ações de saúde bucal, pois não têm atingido a sua população-alvo na prestação de atendimentos odontológicos. Tal asserção torna-se mais relevante no biênio 2010-2011.

Em contrapartida, o IRRE encontrado no município evidencia que o modelo assistencial da APS não tem um perfil mutilador, pois está mais voltado mais para restaurações dos elementos dentários do que para extrações. A média referente aos quatro anos estudados foi 4,7 para as USF e 5,2 para as UBS, sendo que na análise mês a mês houve significância estatística com superioridade das UBS $(\mathrm{p}=0,016)$.

Em 2008, o valor médio do IRRE era de 4,53 para as USF e de 4,31 para as UBS 
(valores próximos), já em 2011 o valor médio deste indicador para as USF subiu para 7,03 (aumento de 63\%) e para as UBS o valor encontrado foi de 6,48 (aumento de 50\%). Estes dados também apontam para um processo de fortalecimento da ESF, assim como evidenciado nos indicadores IEPM e IA.

Entretanto os resultados encontrados neste estudo diferem daqueles expostos por outros autores como, por exemplo, Barros e Chaves (2003) que encontraram em um município do interior da Bahia maior participação dos procedimentos cirúrgicos $(23,3 \%)$ em comparação aos procedimentos restauradores (18,6\%). As visitas domiciliares, a atuação multiprofissional e as reuniões de equipe constituem importantes ferramentas de planejamento da ESF (BALDANI ET AL., 2005). Neste contexto, a Odontologia vem, cada vez mais, se integrando a este cenário que tem alcançado reconhecimento internacional. O IRRE do município estudado demonstra que as mudanças nos modelos assistenciais da APS vêm acontecendo não somente em grandes centros urbanos, mas também em cidades do interior do Brasil.

Esta perspectiva de mudança não pode ser observada na RPCU. A média da APS, considerando USF e UBS, foi de 0,5 , o que significa dizer que a cada primeira consulta odontológica programática são realizadas duas consultas de urgência, ou seja, o dobro. Foi encontrada significância estatística na comparação dos modelos, com superioridade para as UBS $(p=0,002)$, todavia os resultados são bastante insatisfatórios para ambos os modelos.

É relevante considerar que existe uma enorme demanda reprimida de usuários que precisam dos atendimentos de urgência, pois as unidades de saúde da APS ainda não conseguem atender a todas as necessidades odontológicas de sua população adscrita. Geralmente, estas unidades de saúde estão alocadas em comunidades carentes, com populações com a saúde bucal em condições ruins, o que acaba gerando mais demanda para as EqSB e, consequentemente, mais consultas de urgência.

Esta problemática envolve também aspectos socioculturais, pois muitos usuários não valorizam o tratamento odontológico como forma de prevenção de problemas futuros, dando preferência ou buscando atendimento somente nos casos de condições agudas como dores de origem dentária ou traumas. Isto acaba gerando um círculo vicioso, na medida em que a saúde bucal da população continua péssima e as consultas de urgência e emergência se mantêm. Quando não há prevenção e promoção da saúde através de tratamentos odontológicos com abordagem ampla a atuação do CD fica restrita a procedimentos curativos, com isto o modelo assistencial corre o risco de tornar-se voltado para uma prática mutiladora e de reparo de danos. Some-se a isto o fato de que, para as EqSB, práticas de atenção extramuros - como visitas domiciliares e/ ou ações coletivas e de promoção da saúde ainda sejam preteridas em função das ações curativas (DE-CARLI ET AL., 2015).

Segundo Nickel, Lima e Silva (2008), as consultas de urgência são pouco resolutivas e criam mais demanda para novas consultas de urgência, pois o usuário acostuma-se a frequentar a unidade de saúde com esta finalidade. Além disso, geram maior gasto orçamentário, pois é muito mais caro para o sistema de saúde a realização de procedimentos curativos que geralmente necessitam também de encaminhamento para profissionais especialistas.

Vale lembrar que diversas dificuldades estão relacionadas com a RPCU, dentre elas: maior expectativa da população com relação às práticas curativas, insuficiência ou inexistência de serviços especializados e a ausência de técnico em saúde bucal na EqSB, por tratar-se de um profissional com caráter mais preventivo. Todas elas acabam gerando demandas excessivas para as EqSB, que acabam por concentrar seus esforços em ações com maior prioridade, ou seja, 
nos atendimentos de urgência e emergência (FARIAS; MOURA, 2003).

Neste sentido, novos instrumentos de avaliação da saúde bucal no âmbito da APS têm surgido, como o Programa Nacional de Melhoria do Acesso e da Qualidade da Atenção Básica (PMAQ-AB), que se propõe a avaliar a qualidade da APS como uma rede prioritária, como porta de entrada resolutiva para as necessidades de saúde, bem como produzir condições concretas para que ela garanta e coordene a continuidade da atenção. No conjunto de elementos do PMAQ-AB, a saúde bucal é parte integrante e já vem sendo objeto de estudos e investigações que revelam tanto incorporação de práticas quanto permanência de problemas e resistências às mudanças por parte dos profissionais da área (MENDES JÚNIOR; BANDEIRA; TAJRA, 2015; LORENA SOBRINHO ET AL., 2015).

Os ISB verificados pelo PMAQ-AB são: média da ação coletiva de escovação dental supervisionada; cobertura de primeira consulta odontológica programática; cobertura de primeira consulta de atendimento odontológico à gestante; razão entre tratamentos concluídos versus primeiras consultas odontológicas programáticas; média de atendimentos de urgência odontológica por habitante; média de instalações de próteses dentárias e taxa de incidência de alterações da mucosa oral (CASOTTI ET AL., 2014). Ou seja, é possível estabelecer uma importante coincidência entre os ISB utilizados nesta pesquisa com aqueles abordados pelo PMAQ-AB.

Casotti et al. (2014) realizaram um estudo com o objetivo de identificar as características da oferta de serviços de odontologia acessados pelos usuários por meio do PMAQ-AB quanto aos atributos do acesso de primeiro contato e integralidade. Os resultados obtidos indicam que $45,1 \%$ dos usuários consegue marcar consulta com o dentista, porém o acesso/tempo de espera nas especialidades ainda se constitui em problema da rede de atenção. A garantia de continuidade de tratamento é menor nas regiões Norte e Nordeste, quando comparadas às demais regiões brasileiras.

Quanto às limitações do SIA-SUS, cabe destacar que os dados desse sistema são relativos aos procedimentos realizados, portanto não permitem investigação de perfis de morbidade. Outro aspecto importante está relacionado com o tempo destinado pelos trabalhadores da saúde para o registro diário dos procedimentos realizados e que pode ser dispendioso dentro de uma rotina de trabalho atribulada.

Apesar das limitações acima explicitadas é imprescindível a utilização dos SIs como fonte de dados para estudos na área da saúde. Neste sentido, os ISB se mostram eficientes instrumentos de análise quantitativa, pois transformam dados numéricos em informações mais fáceis de serem interpretadas e comparadas (CARNUT; FIGUEIREDO; GOES, 2010).

A valorização dos SIs é discussão recorrente na grande maioria dos municípios e regiões do Brasil. Enquanto não houver incorporação da utilização destas ferramentas no processo de trabalho corre-se o risco de atuar somente em livre demanda de atendimento, sem qualquer planejamento ou diagnóstico do território. A finalidade destes SIs não deve ficar restrita somente a previsões orçamentárias, mas sim ser direcionada para o fortalecimento das equipes e a construção de uma prática com maiores resolubilidade, equidade, integralidade e universalidade (FERNANDES; PERES, 2005).

\section{Conclusões}

No momento em que a Política Nacional de Saúde Bucal completa uma década, estudos de avaliação dos resultados das práticas e ações desenvolvidas no âmbito da APS são fundamentais para a construção de subsídios que ponham em análise os impactos, 
as potencialidades e fragilidades presentes nesta e noutras dimensões da política de saúde em que se coloca a própria sustentabilidade do SUS.

Este estudo permitiu apontar, em um cenário significativo, quanto há por avançar na consolidação de um modelo assistencial que integre práticas curativas e práticas de prevenção/promoção. Para o alcance deste objetivo, trata-se de combinar uma reorientação das práticas de saúde bucal com a expansão da atenção capilarizada pela ESF. Integração em que a saúde bucal, em particular, expressa um forte apelo simbólico - um país sorridente: o sorriso com uma aparência bucal íntegra seria uma boa imagem da garantia do direito a sorrir sem constrangimentos.

Diferenças significativas $(\mathrm{p}<0,05)$ foram observadas para IRRE e RPCU. Pode-se concluir que, embora as UBS tenham apresentado melhor desempenho do que as USF no município estudado, os resultados também indicaram um processo de fortalecimento da ESF. IEPM, IA e RPCU demonstraram resultados insatisfatórios, apontando para dificuldades de realização de ações de prevenção e promoção da saúde, além de obstáculos para atingir o público-alvo e os atendimentos odontológicos concentrados em urgências e procedimentos curativos. IR e IRRE apresentaram os melhores

\section{Referências}

\footnotetext{
AQUILANTE, A. G.; ACIOLE, G. G. Oral health care after the National Policy on Oral Health - "Smiling Brazil”: a case study. Ciênc. saúde colet., Rio de Janeiro, v. 20, n. 1, p. 239-248, jan. 2015. Disponível em: <http:// www.scielosp.org/scielo.php?script=sci_arttext \&pid=S1413-81232015000100239\&lng=en \&nrm=iso . Acesso em: 4 mar. 2015.
}

resultados, indicando alta produtividade em termos de atendimentos odontológicos na atenção básica e um modelo assistencial voltado para práticas conservadoras.

Apesar do processo de fortalecimento da ESF, o modelo assistencial representado pelas UBS ainda se mostra mais efetivo em alguns aspectos da saúde bucal no serviço público, aspectos pautados principalmente com a relação restauração/extração e com atendimentos menos voltados para urgência/emergência.

Ainda que se trate do estudo de um município, com resultados que devem ser considerados dentro do limite metodológico, evidenciou-se um achado preocupante no seu potencial de significar um cenário não ocasional em termos de permanência ou persistência de um modelo assistencial centrado nos procedimentos mutiladores, tão difundido em décadas pré-SUS. Afinal, os aspectos insatisfatórios, que aparecem para ambos os modelos, são obstáculos a serem vencidos pela odontologia da APS, tais como: a efetiva realização de ações de prevenção e promoção da saúde, além da capacidade de atingir eficazmente a população-alvo modificando seus valores de uso a respeito dos dentes e sua relação com a saúde mais geral do corpo, de modo a impactar o imaginário ainda forte de que 'os dentes podem ser substituídos'.
BALDANI, M. H. et al. A inclusão da odontologia no Programa Saúde da Família no Estado do Paraná, Brasil. Cadernos Saúde Coletiva, Rio de Janeiro, v. 21, n. 4, p. 1026-1035, 2005.

BARROS, S. G.; CHAVES, S. C. L. A utilização do Sistema de Informações Ambulatoriais (SIA-SUS) 
como instrumento para caracterização das ações de saúde bucal. Epidemiologia e Serviços de Saúde, Brasília, DF, v. 12, n. 1, p. 41-51, 2003.

CARNUT, L.; FIGUEIREDO, N.; GOES, P. S. A. Saúde bucal na atenção primária brasileira: em busca de um sistema de informação em saúde. Journal Management Primary Health Care, Olinda, v. 1, n. 1, p. 8-13, 2010.

CASOTTI, E. et al. Atenção em Saúde Bucal no Brasil: uma análise a partir da Avaliação Externa do PMAQAB. Saúde em Debate, Rio de Janeiro, v. 38, n. esp., p. 140-157, 2014.

CELESTE, R. K. et al. Séries de procedimentos odontológicos realizados nos serviços públicos brasileiros, 1994-2007. Ciência Saúde Coletiva, Rio de Janeiro, v. 16, n. 11, p. 4523-4532, 2011.

DE-CARLI, A. D. et al. Visita domiciliar e cuidado domiciliar na atenção básica: um olhar sobre a saúde bucal. Saúde Debate, Rio de Janeiro, v. 39, n. 105, p. 431-450, 2015.

EMMI, D. T.; BARROSO, R. F. Evaluation of oral health actions in the Family Health Program in the Mosqueiro district, Pará State, Brazil. Ciência Saúde Coletiva, Rio de Janeiro, v. 13, n. 1, p. 35-41, 2008.

FARIAS, M. A. V.; MOURA, E. R. F. Saúde Bucal no contexto do Programa Saúde da Família do município de Iracema, no Ceará. Revista de Odontologia da UNESP, Araraquara, v. 32, n. 2, p. 131-137, 2003.

FERNANDES, L. S.; PERES, M. A. Associação entre Atenção Básica em Saúde Bucal e indicadores socioeconômicos municipais. Revista Saúde Pública, São Paulo, v. 39, n. 6, p. 930-936, 2005.

LOIVOS, C. et al. Acesso às ações de saúde bucal versus indicadores do SIAB/SIASUS: um diálogo possível e necessário. Revista Brasileira de Odontologia, Rio de Janeiro, v. 66, n. 2, p. 263-269, 2006.

LORENA SOBRINHO, J. E. et al. Acesso e qualidade: avaliação das equipes de saúde bucal participantes do
PMAQ-AB 2012 em Pernambuco. Saúde Debate, Rio de Janeiro, v. 39, n. 104, p. 136-146, 2015.

MENDES JÚNIOR, F. I. B.; BANDEIRA, M. A. M.; TAJRA, F. S. Percepção dos profissionais quanto a pertinência dos indicadores de saúde bucal em uma metrópole do Nordeste brasileiro. Saúde Debate, Rio de Janeiro, v. 39, n. 104, p. 147-158, 2015.

NARVAI, P. C. Avaliação das ações em saúde bucal. São Paulo: Secretaria de Estado da Saúde de São Paulo: Rio de Janeiro: Oficina Saúde Bucal no SUS, 1996.

NICKEL, D. A.; LIMA, F. G.; SILVA, B. B. Modelos assistenciais em saúde bucal no Brasil. Cadernos Saúde Coletiva, Rio de Janeiro, v. 24, n. 2, p. 241-46, 2008.

PEREIRA, C. R. S. et al. Impacto da Estratégia Saúde da Família sobre indicadores de saúde bucal: análise em municípios do Nordeste brasileiro com mais de 100 mil habitantes. Cad. Saúde Pública, Rio de Janeiro, v. 28, n. 3, p. 449-462, 2012.

ROCHA, R. A. C. P.; GOES, P. S. Comparison of access to Oral Health Services between areas covered and not covered by the Family Health Program in Campina Grande, Paraíba State, Brazil. Cad. Saúde Pública, Rio de Janeiro, v. 24, n. 12, p. 2871-2880, 2008.

SOUZA, T. M. S.; RONCALLI, A. G. Saúde bucal no Programa Saúde da Família: uma avaliação do modelo assistencial. Cad. Saúde Pública, Rio de Janeiro, v. 23, n. 11, p. 2727-2739, 2007.

VOLPATO, L. E. R.; SCATENA, J. H. Análise da política de saúde bucal do município de Cuiabá, estado de Mato Grosso, Brasil, a partir do banco de dados do Sistema de Informações Ambulatoriais do Sistema Único de Saúde (SIA-SUS). Epidemiologia e Serviços de Saúde, Brasília, DF, v. 15, n. 2, p. 47-55, 2006.

Recebido para publicação em agosto de 2015

Versão final em dezembro de 2015

Conflito de interesses: inexistente

Suporte financeiro: não houve 